\title{
Cascade Effects of Load Shedding in Coupled Networks
}

\author{
Sotharith Tauch, William Liu and Russel Pears \\ School of Computing and Mathematical Sciences \\ Auckland University of Technology \\ Auckland, New Zealand \\ Email: \{sotharith.tauch, william.liu, russel.pears\}@aut.ac.nz
}

\begin{abstract}
Intricate webs of interlinked critical infrastructures such as electrical grid, telecommunication, and transportation are essential for the minimal functioning of contemporary societies and economies. Advances in Information and Communication Technology (ICT) underpin the increasing interconnectivity of these systems which created new vulnerabilities that can be seriously affected by hardware failure, link cut, human error, natural disaster, physical-attacks and cyber-attacks. Failure of a fraction on nodes may lead to failure of dependent nodes in the other networks. Therefore, the main objective of this paper is to investigate the cascades phenomena caused by load shedding between two interconnected networks using Bak-Tang-Wiesenfeld sandpile modeling. We have found that, large avalanche occurred when node degree and/interconnectivity link become dense. In addition, the coupled random-regular networks have been found to be more robust than the coupled Erdös-Rényi networks. However, coupled random-regular networks are vulnerable to random attack and coupled Erdös-Rényi networks are vulnerable to target attack due to the degree distribution.
\end{abstract}

Keywords-cascading effects; interdependence; coupled networks; network robustness; complex networks; Sandpile modelling.

\section{INTRODUCTION}

The emphasis of cascading failures phenomena in complex networks has become an important aspect in trying to understand the fragility of the network or networks, so as to make the networks more robust and secure. Real-world examples of complex network are the Internet, electrical power grids, social networks, and telecommunication network [1-3]. Most of critical infrastructures todays are interconnected which capable of sharing information, load, and capacity. Interconnectivity allows building sophisticated network of networks; however, the complexity of these networks is arising which creates vulnerability against cascading failures. An example of some critical infrastructures is electrical Smart Grid network. Failures in electrical system can cause major disruption to the Internet, transportation, and our day to day life. A series of failure or blackout of an entire network can causes by failure of a small fraction of the network. Real-world events with series of blackout have already happened in the past and continuing to happen today. On July and August 1996, two power blackout events in west America which led to 11 states out of power [4]. The largest blackout in the history of the United State happened in August 2003, the event triggered in the power grid of the U.S. and Canada [5]. On the $20^{\text {th }}$ February 1998, a major blackout in Auckland CBD caused by failures of four power lines which left the entire city without power [6]. April 2012, a power outage at the KiwiRail Train Control Centre in Wellington caused by failure of the uninterrupted power supply system led to a shutdown of railway systems in Auckland during rush hours [7]. These events exhibit the phenomena of failures triggered by an event and cascade to other interconnected networks. In this study we investigate cascading phenomena in coupled interactive networks in term of load shedding from one node to another node and eventually cascades to the neighboring interconnected network.

This paper is organized as follows. In section II, we review the related work in this area. In section III, the system model is introduced by presenting an overview of sandpile dynamics model and the interactive network topology. The extensive results from simulation are analyzed and discussed in Section IV. The final conclusions are drawn in session $\mathrm{V}$ and also the future works are identified.

\section{LITERATURE REVIEW}

In interconnected networks, failures of a fraction of nodes may lead to a series of failures cascade throughout the entire network. A framework was developed by Buldyrev et al. [8] to understand the resiliency of interdependent networks subjected to failures cascade from one network to another. This framework contains a model consists of two identical networks interconnected by dependency links. Nodes are randomly connected and the degree distributions are, $P_{A}(k)$ and $P_{B}(k)$. Percolation method was adopted in identifying the existence of giant connected component. The results show the recursive process of cascading failures happened when a critical portion of the nodes in one network failed, which leads the system to undertakes first order phase transition.

Buldyrev et al. [9] introduced a new model known as correspondently coupled network (CCNs). This model consists of two mutually dependent networks with identical number of nodes and degree distributions. Mutual percolation framework was used in identifying the strongest possible relationship between the two networks and investigating the broadness of 
the degree distribution. Results show that if the second instant of the degree distribution is limited, CCNs disintegrate in a cascade of failures via a first-order transition. In addition, if the degree distribution becomes broader, CCNs becomes more robust. Parshani, et al. [10] proposed another model of two networks where only a fraction of nodes in one network depends on a fraction of nodes in the other network. This model was formalized based on a real Smart Grid network. This model is limiting to a condition that all nodes in each network are randomly connected with degree distribution $P(k)$. Percolation framework again used to identify phase change in percolation transitions after reducing the coupling strength in interdependent networks. Simulations were conducted on two different types of coupled network, Scale-Free (SF) networks and Erdös-Rényi (ER) networks.

Shao, et al. [11] proposed a model based on multiple support-dependence relations. Two networks interconnected and each node in one network is depending on multiple nodes in the other network. This model was defined in accordance to two conditions such that, for a node in one network to be functional it must (i) have at least a functional support node from the other network and also (ii) it must belong to the giant component of functional nodes within its own network. Numerical simulations have been conducted thoroughly on Erdös-Rényi (ER) and Scale-Free (SF) networks using percolation method. The result shows that, multi supportdependent relations model disintegrated at the first order phase transition. Gao, et al. [12] proposed a new theory known as a network of networks, which developed based on percolation approach which is a general analytical framework in the study of percolation of $n$ interdependent networks. This model consists of more than two interdependent networks, known as network of networks (NONs). The result shows that, of any network size starting from $n \geq 2$, the phase transition becomes first-order transition and cascade of failures is as expected become visible for strong coupling.

The literatures above were focusing on the cascading failures in interdependent networks in the cases of node and links failures. However, our target in this study is to investigate the cascading failures phenomena in coupled networks caused by load shedding when a node becomes unstable. If a node becomes unstable, its load will shed to the neighboring nodes.

\section{THE MOdELS FOR DYNAMIC LOAD SHEDDING}

In this section we introduce the related mathematical models used in this study to quantify the cascades. We used BTW sandpile dynamics to model load shedding phenomena and apply this model to coupled networks model which will be constructed using random-regular networks and Erdos Renyi networks. These models help to evaluate the behavior of load shedding in coupled interactive networks based on avalanche size and probability of avalanche size distribution. Details of models and avalanche size distribution are described below.

\section{A. BTW Sandpile Dynamics Model}

In 1987 and 1988, Bak, et al. introduced a model known as Bak-Tang-Wiesenfeld (BTW) sandpile dynamics which named after the three authors. This model was realized from their studies in self-organized criticality in dynamical systems. BTW is a well-known model which has been used in many studies of cascades phenomena that exhibit self-organized criticality [13,
14]. In this study we adopted this model for the purpose of validating the property of load shedding and the avalanche size distribution among two interacting networks. The concept of this model was derived from real sand pile behavior. In this study we adopted the 2-dimensional lattices concept and the process of this model is introduced by dropping grains of sand uniformly at random on network nodes each of which has an individual threshold. Throughout this process, when a node exceeds its capacity or threshold, this node will begin to topple or shed gains of sand to its neighboring nodes. At this stage if the neighboring nodes become unstable or failure, again grains of sand will start to topple or shed to the other neighboring nodes until load does not exceed any other node capacity. However, upon dropping another grain of sand, the toppling or shedding process might begin again.

In this study, we assume that grains of sand represent as load and threshold represented as degree or capacities of each node. What we are interesting is the probability of avalanche size distribution, which is the chance that the load might topple large number of network nodes. In 2-dimensional finite lattices with open boundaries condition, some sands are lost when arriving at the boundaries which are naturally equivalent to delete grains of sand independently with probability $f$. The probability $f$ is also known as dissipation rate [15]. Consider sandpile model of 2-dimentional finite $L \times L$ networks, every node in each network is denoted by $Z_{i}$ where:

$$
Z_{i}=\mathrm{Z}(\mathrm{x}, \mathrm{y})=0,1,2,3, \ldots \quad x, y=1,2, \ldots, L .
$$

At initial state $Z_{i} \ll \mathrm{K}$, when there is no grain of sand being dropped. $K$ is the threshold of every node. Upon dropping grains of sand uniformly at random, at some stages when a grain of sand is added to a node with the capacity equal to its threshold $Z_{i}=K$, a toppling event occur that might leads to a whole series of topplings. A toppling event is represented by $Z_{i} \rightarrow Z_{i}+1$, when $Z_{i}=K$. The toppling events will continue until it reaches a stable state, where there is no other node topple.

1) Avalanche size distribution: In this study, we focus on the probability of avalanche size distribution $D(s)$, where $D(s)$ is the chance that an avalanche begun in either network A or network B causing s number of nodes to topple. The avalanche size distribution can be derived from the following probability distribution of branching process $P(s, p)$ [16].

Let $f(x, p)$ be the generating function of $\mathrm{P}(s, p)$, we have:

$f(x, p)=\sum_{s} P(s, p) x^{s}$

By taking derivative of equation (1), we have:

$$
P(s, p)=\left.\frac{1}{s !} \frac{\partial^{s} f(x, p)}{\partial x^{s}}\right|_{x=0}, n, p \text { are fixed. }
$$

The equation (1) can be simplified as follow:

$f(x, p)=x(1-p)+x p f^{2}(x, p)=\frac{1-\sqrt{\left(1-4 x^{2} p(1-p)\right)}}{2 x p}$

Equation (3) can be solved in the power of $x^{2}$ for s and $\mathrm{k}$ :

$\frac{1}{p}[4 p(1-p)]^{k} \frac{\left(x^{2}\right)^{k}}{x}=\frac{1}{p}[4 p(1-p)]^{k} x^{2 k-1}$

Equation (4) is correlated to equation (2), which we get: $s=2 k-1$ and $k=\frac{s+1}{2}$ 
In this case:

$$
\begin{gathered}
P(s, p) \sim \frac{1}{p} \sqrt{4 p(1-p)}[4 p(1-p)]^{s / 2} \sim e^{-s / s_{c}(p)} \\
a^{\frac{s}{2}}=e^{\ln \left(a^{\frac{s}{2}}\right)}=e^{-s\left(\frac{\ln (a)}{-2}\right)}, \quad a=4 p(1-p), \\
s_{c}(p)=\frac{-2}{\ln [4 p(1-p)]}, \quad \lim _{p \rightarrow 1 / 2} s_{c}(p) \rightarrow \infty . \\
\text { When } p=\frac{1}{2}, 4 p(1-p)=1, f(x, p)=\frac{\left.1-\sqrt{\left(1-x^{2}\right.}\right)}{2 x p} \\
\sqrt{1-x^{2}}=\sum_{k=0}^{\infty} \frac{\frac{1}{2}\left(\frac{1}{2}-1\right)\left(\frac{1}{2}-2\right) \ldots\left(\frac{1}{2}-k+1\right)}{k !}\left(-x^{2}\right)^{k} \\
P_{c}(k)=P\left(s=2 k-1, p=\frac{1}{2}\right) \\
\quad=\frac{\frac{1}{2}\left(\frac{1}{2}-1\right)\left(\frac{1}{2}-2\right) \ldots\left(\frac{1}{2}-k+1\right)}{k !}(-1)^{k} \\
\text { Thus } P_{c}(K+1)=\frac{\frac{1}{2}-k}{k+1}(-1) P_{c}(k)=\frac{1-1 /(2 k)}{1+1 / k} P_{c}(k)
\end{gathered}
$$

In the limit of large $k$, we have $k=(s+1) / 2$,

where $1 /(1+1 / k)=1 / 1-k$,

$$
P_{c}(k+1) \approx\left[1-\frac{1}{2 k}\right]\left[1-\frac{1}{k}\right] P_{c}(k) \approx\left[1-\frac{3}{2 k}\right] P_{c}(k)
$$

This asymptotic relation leads to

$$
\frac{P_{c}(k+1)-P_{c}(k)}{1}=\frac{-3}{2 k} P_{c}(k), \quad \frac{\partial P_{c}(k)}{\partial k}=\frac{-3}{2 k} P_{c}(k)
$$

Thus, we have the solution

$$
P_{c}(k) \sim k^{-3 / 2}, \quad D(s)=P_{c}(s) \sim s^{-3 / 2}, \quad \alpha_{s}=3 / 2
$$

for large $k, s$, since $s=2 k-1$

\section{B. Topologies of Coupled Networks}

This study focused on two interconnected networks labeled as A and B. Both networks are constructed based on two graph models. In the first model, the network topology is constructed using random-regular graphs. These two networks are sparsely interconnected at random using Bernoulli-coupling model [15]. Nodes in each network are randomly connected based on a given node degree. In the second model, Edös Rényi random graph is used to construct the network topology with the same configuration as in the first model. Nodes in each network are randomly connected based on a given values of node degrees. Fig. 1 shows an example of the topology of interacting networks.

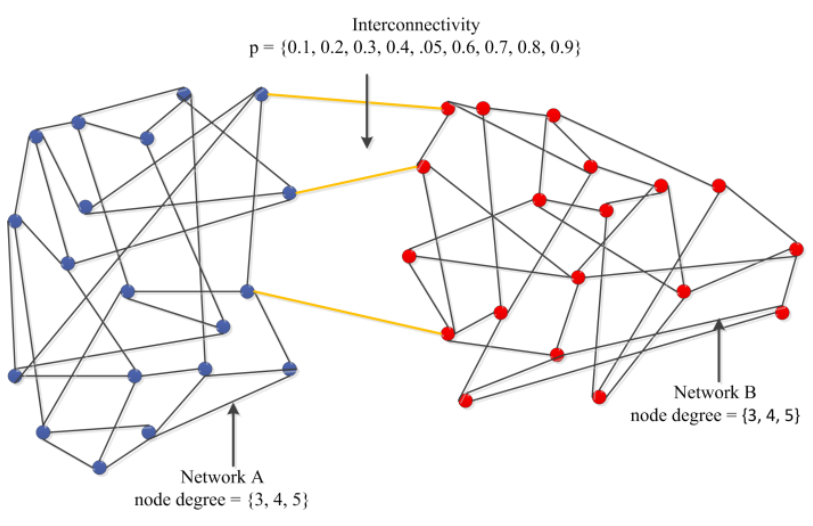

Figure 1. Example of a coupled networks topology.

In each network, the connectivity between nodes is based on given values of node degree $\left(I_{a}=I_{b}=\{3,4,5\}\right)$. The yellow links represented as the interconnectivity between the two networks based on Bernoulli-Coupling model. In this case, the $p$ value in the graph represents as interconnectivity for both networks. Each network has probability of degree distribution denoted by $P_{a}\left(I_{a}, p\right), P_{a}\left(I_{a}, 1-p\right)$ and $P_{b}\left(p, I_{a}\right), P_{b}\left(1-p, I_{a}\right)$ for network $A$ and for network $B$ respectively.

1) Random-Regular Graph: is a graph model represents by $G(n, m)$, where $n$ is the number of vertices which can be label as $n=\{1,2,3, \ldots ., n\}$ and $m$ is the degree (number of edge) for every vertex. The average degree of $\mathrm{RR}$ network is $\langle k\rangle=$ $2 m / n[17]$.

2) Erdös Rényi (ER) Graph: is also known as Poisson random graph which was first studied by Edös and Rényi [3]. ER random graph is represented by $G(n, p)$ where $n$ is the number of vertices and $p$ is the probability of having edges between vertices. An important property of ER random graph is the degree distribution represented by $P(k)$ the probability of a node being connected to exactly $k$ other nodes [18].

$$
P(k)=\left(\begin{array}{c}
n-1 \\
k
\end{array}\right) p^{k}(1-p)^{n-1-k}=e^{-c} \frac{\langle k\rangle^{k}}{k !}
$$

Equaiton (6) shows that ER has Poisson degree distribution.

\section{Simulation StUdies}

To understand cascade effects in coupled ER and RR networks, we simulated the effects of load shedding where number of nodes and node degree in each network are identical. As mentioned in section III above, two different graph models will be used in constructing coupled networks models. The simulation is divided in two different sets. In the first set, random-regular graph model is being used and ER random graph model is being used in the second set. The simulation results will be analyzed in the discussion sections below. From the results, we are most interested in the probability of avalanche size distribution $D(s)$ and the avalanche size $s$. This simulation study is conducted by applying BTW sandpile dynamics model into python based NetworkX tool [19]. The parameters in the simulation are number of nodes (set to 500) of each network, fix dissipation rate $f=0.1$, interconnectivity $p=\{0.1,0.2,0.3, \ldots, 0.9\}$ and node degree $\left.I_{a}=I_{b}=\{3,4,5\}\right)$.

\section{A. Impact of Degree Distribution in RR and ER Coupled Networks Models}

In this section, we will be discussing the probability degree distributions gathered from the simulation. First of all, coupled random-regular (RR) networks will be discussed. In term of degree distribution, Fig. 2 shows the distribution of node degree with given node degree $I_{a}=I_{b}=\{3,5\}$ and BernoulliCoupling of interconnectivity link. Referring to Fig. 2A and Fig. 2B, each node in RR networks model has almost identical number of node degree. However, the maximum degree obtained from the simulation is greater than the given node degree. This is due to the fact that, some nodes in one network have an additional interconnectivity link to another network. In this case the probability distribution of network $A$ is denoted by the joint probabilities $P_{a}\left(I_{a}, p\right)$ - a node has an interconnectivity link and $P_{a}\left(I_{a}, 1-p\right)-$ a node has no interconnectivity link. Analogously, the same probability distribution applies to network B. This method is known as 
Bernoulli-Coupling. As shown in Fig. 2A and Fig. 2B, when $p$ increases to 0.9 , almost every node has the degree of 4 , which means almost every node has an interconnectivity link.
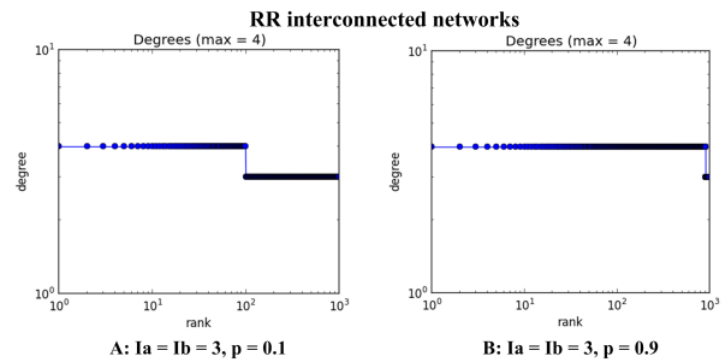

ER interconnected networks
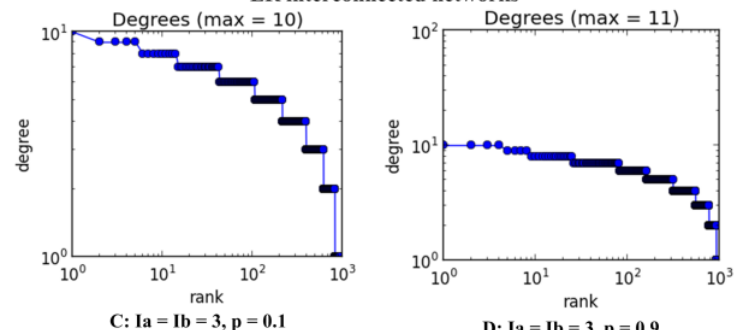

Figure 2. RR and ER Networks with $\mathrm{I}_{a}=\mathrm{I}_{b}=3, \mathrm{p}=\{0.1,0.9\}$

The degree distribution in RR network is uniformly distributed based on a given number of node degree. On the other hand, the degree distribution in ER networks is distributed based on the probability of having edges between a given numbers of nodes. Fig. 2C and Fig. 2D show the degree distribution of ER networks with given node degree of 3 and interconnectivity values of 0.1 and 0.9 respectively. In comparison to the degree in RR networks, the maximum degree in ER is greater than RR and the degree of most nodes are not identical.

\section{B. Impact of Average Node Degree and Interconnectivity on Avalanche Size in RR Networks}

In this section we conducted simulations on two identical RR networks with fix values of node degree $\left(I_{a}=I_{b}=\right.$ $\{3,4,5\})$ and increasing interconnectivity degree from $p=\{0.1$, $0.5,0.9\}$ for every fix value of $I_{a}$ and $I_{b}$ accordingly.
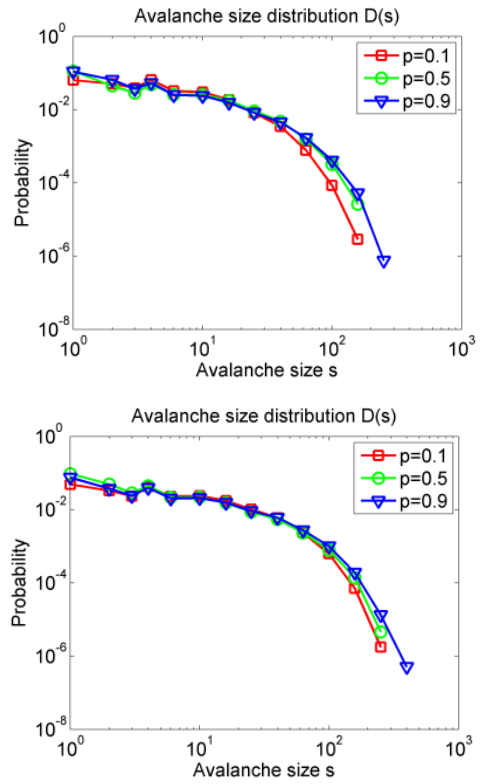

(A)

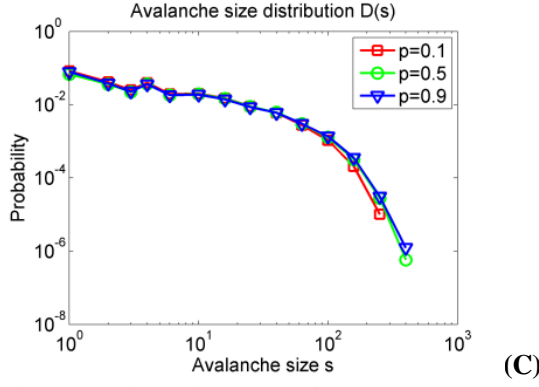

Figure 3. Fix node degree of 3, 4, and 5 respectively

According to Fig. 3(A), Fig. 3(B), and Fig. 3(C), the diagrams show exponentially decrease in all three cases which indicated that the probability of avalanche size $D(s)$ decreases when avalanche size $s$ increases. When $p$ increased in the range of $\{0.1,0.5,0.9\}$, the avalanche size increased significantly. The smallest avalanche size occurred at $p=0.1$ and as $p$ increases to 0.9 , large avalanche size occurred. However, the chance of this large avalanche size to happen is lower than at $p=0.1$.

In the next comparative analysis, the value of $I_{a}$ and $I_{b}$ will be changing in the range of $\{3,4,5\}$ with fix values of interconnectivity $p=\{0.1$ and 0.9$\}$.

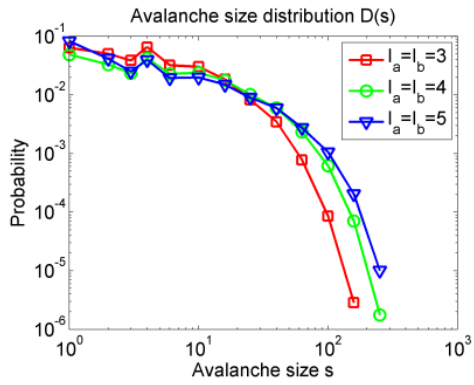

(A)

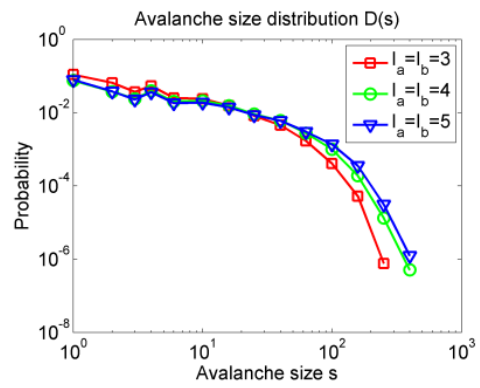

(B)

Figure 4. Fix interconnectivity degree of $p=\{0.1$ and 0.9$\}$ respectively

The diagrams in Fig. 4(A) and Fig. 4(B) show exponentially decrease in both cases. By increasing the value of $I_{a}$ and $I_{b}$, the probability of avalanche size distribution $D(s)$ decreased exponentially. However, the avalanche size $s$ increases significantly. The event of small avalanche occurred when node degree $I_{a}=I_{b}=3$ for every $p$ value and the largest avalanche occurred when the node degree becomes dense for every $p$ value. This indicated that, increases in average node degree can enforce avalanche size to also increase. In addition, when the node degree is increasingly dense, the probability of avalanche size distribution $D(s)$ has the highest value. In other word, the chance of having large avalanche size is increasing.

The results above indicated that, by coupling more links between the two networks, the avalanche will be cascading across the entire network and the chance of having large 
avalanche is very low; however, a significant number of nodes will fail if this is to happen. On the other hand, by coupling more links within each network, the avalanche is increasing as well as the chance of having large avalanche. The reason behind this phenomena is the degree distribution. When the interconnectivity between the two networks becomes dense, both networks become completely integrated because almost every node in one network is connected to almost every other node in the neighbouring network. On the other hand, when the degree within each network becomes dense, the chance of load topple significant number of nodes in one network and quickly cascade to the other network is increasing. In addition, node degree in RR coupled networks is uniformly distributed which mean every node has almost identical degree. So in this case, every node are identically important to the network in term of degree distribution.

\section{Impact of Average Node Degree and Interconnectivity on Avalanche Size in ER Networks}

We conducted the second set of simulations using coupled ER networks model with fix node degree $\left(I_{a}=I_{b}=\{3,4,5\}\right)$ and changing the interconnectivity $p=\{0.1,0.5,0.9\}$. The graphs in Fig. 5(A), Fig. 5(B), and Fig. 5(C), show exponentially decrease in all three cases. Thus, the probability of avalanche size distribution $D(s)$ decreases with the increase in avalanche size.

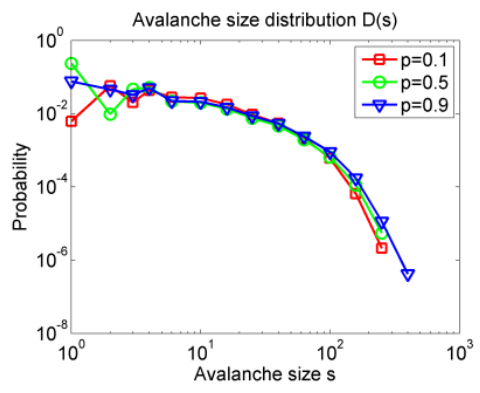

(A)

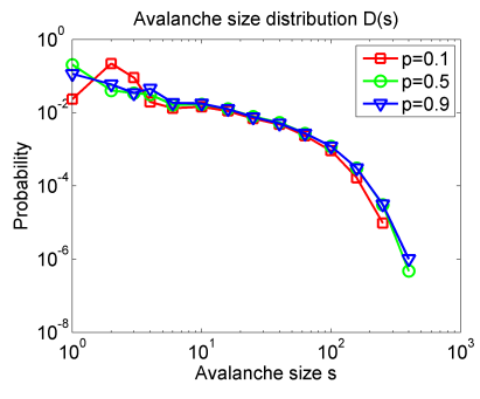

(B)

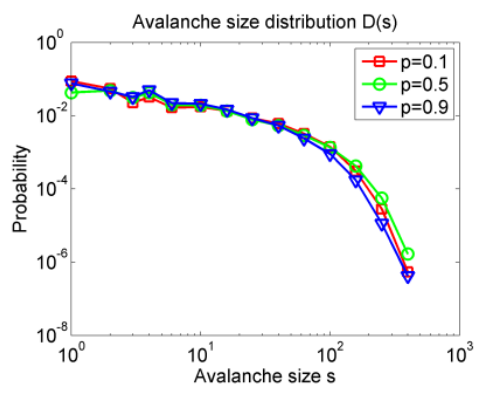

(C)

Figure 5. Fix node degree of 3, 4, and 5 respectively

When interconnectivity increases with the values of $p=$ $\{0.1,0.5,0.9\}$, the avalanche shows significantly increases in size. However, there is an exception in Fig. 5(C), when average node degree becomes dense, the largest avalanche occurred for every $p$ value. The lowest probability of avalanche size distribution occurred only at $p=0.9$. From these three cases, the simulation results exhibited an interesting behaviour when node degree becomes dense. In this case, increasing interconnectivity does not increase the avalanche size when node degree becomes dense. Increasing node degree with the values of $I_{a}=I_{b}=\{3,4,5\}$ at each fix average external degree of $p=\{0.1,0.9\}$ is presenting next. The diagrams in Fig. 6(A) and Fig. 6(B), again show exponentially decrease for all two cases.

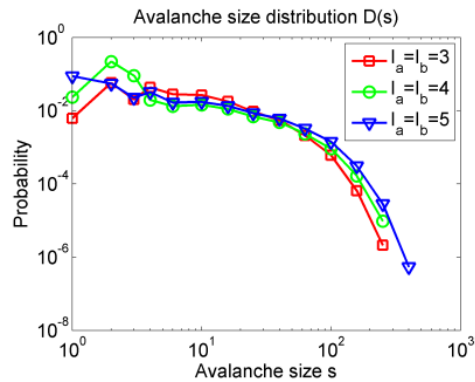

(A)

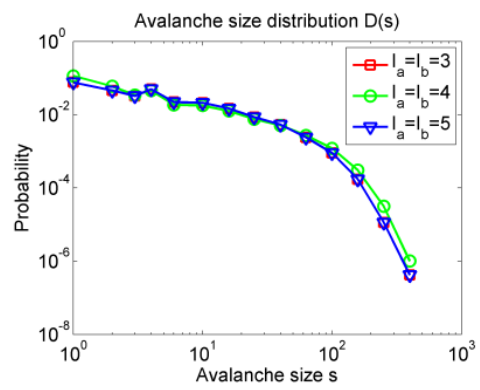

(B)

Figure 6. Fix interconnectivity degree of $p=\{0.1$ and 0.9$\}$ respectively

Fig. 6(A) above shows, at $p=0.1$ when node degree increasing from $I_{a}=I_{b}=\{3,4,5\}$, the avalanche size increases significantly. This indicated that, the largest avalanche size occurred when node degree increases. However, Fig. 6(B) shows an interesting result at which the largest avalanche occurred for every given values of node degree when $p=0.9$. This indicated that, the increasing in node degree does not increase the avalanche size when the interconnectivity becomes dense. The probability of avalanche size distribution is small when $I_{a}=I_{b}=5$.

From the above two cases, the avalanche size shows significant increase only when the node degree is less than 5. However, when interconnectivity degree becomes dense, the avalanche sizes shows identically large even with small node degree. In this case, by coupling more links either between both network or in each individual network, the avalanche size is increasing and the probability of avalanche size distribution is decreasing. Again the reason behind this phenomena is the degree distribution. Node degree in ER coupled networks is random distributed which mean some nodes have higher degree than other nodes. So in this case, the nodes with higher degree can be identified as the hubs of the network which is very critical if these nodes fail. If the hubs of the network fail, the number of topple nodes is also high because load on failed nodes will shed to every neighbouring nodes which might again cause the neighboring nodes to topple. 


\section{Comparison between RR and ER coupled networks}

In previous sections, we analyzed RR and ER individually. To understand the different behaviors between RR and ER networks, we will be comparing the avalanche size and the probability of avalanche size distribution between these two types of networks and we will also identify the vulnerability of these networks toward random and target attacks.
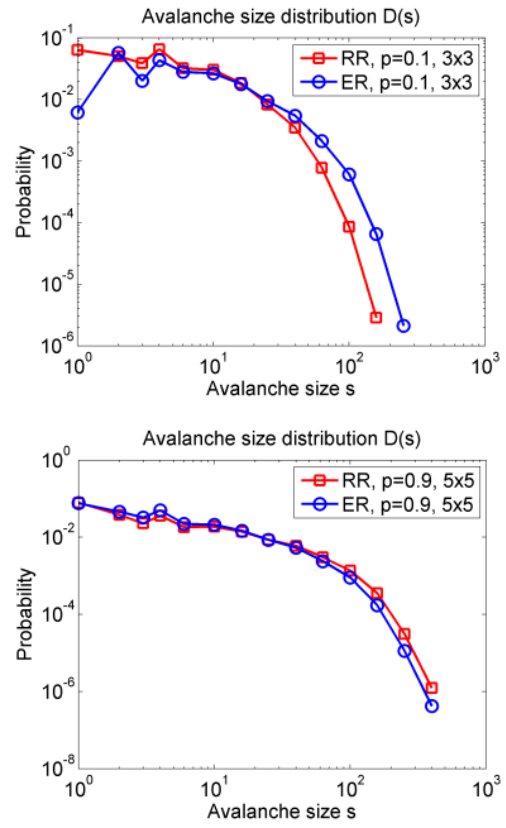

(B)

Figure 7. Compare RR and ER with small and large degree respectively

When nodes in each network and between the networks are sparsely connected, Fig. 7(A) shows that, ER exhibit larger avalanche size than RR networks. This indicated that, at small average degree RR is more robust than ER networks. As the degree become dense, Fig. 7(B) shows an interesting behaviour. In this case, both networks are completely integrated into one network. From Fig. 7(B), both RR and ER coupled networks have identically large avalanche size. However, the chance of having this large avalanche size in RR networks is slightly smaller than ER networks. In correlation to the degree distribution every node in RR networks is almost equally important which lead to vulnerability against random attack. On the other hand, some nodes in ER networks are the hubs of the networks. So if the attack is targeted on those hubs, large avalanche size will occur.

\section{CONCLUSION}

In this paper we investigated load shedding phenomena in interacting networks by applying Bak-Tang-Wiesenfeld sandpile model in the simulation with two cases of randomregular (RR) networks and Edös Rényi (ER) networks. Our main focus is to study the correlation between the degree distribution, the avalanche size, and the probability of avalanche size distribution. We have found that, RR coupled networks undergone large avalanche when increased either node degree or interconnectivity. By increasing interconnectivity, the probability of avalanche size distribution is decreasing. However, when increasing node degree, the probability of avalanche size distribution is also increasing.
On the other hand, increasing node degree or interconnectivity in coupled ER networks, the probability of avalanche size distribution is decreasing but the networks undergone large cascade. In term of vulnerability of coupled random-regular network and coupled Erdös-Rényi networks, we have identified that coupled ER networks are more vulnerable to target attack and coupled RR networks are more vulnerable to random attack.

In future work, we are targeting to analytically model more complex coupled networks such as scale-free networks from the reality. In addition, we need to identify the sensitive network structural factors which have significant impact on the cascade effects which can be used to quantify and control the cascades, so as to further develop new solutions to tackle the challenging robustness problems in coupled networks.

\section{REFERENCES}

[1] S. H. Strogatz, "Exploring complex networks," Nature, pp. 268-276, 2001.

[2] X. F. Wang and G. Chen, "Complex networks: small-world, scale-free and beyond," Circuits and Systems Magazine, IEEE, pp. 6-20, 2003.

[3] L. Zhao, K. Park, Y.-C. Lai, and T. Cupertino, "Attack induced cascading breakdown in complex networks," Journal of the Brazilian Computer Society, vol. 13, pp. 67-76, 2007/09/01 2007.

[4] K. Sun and Z.-X. Han, "Analysis and Comparison on Several Kinds of Models of Cascading Failure in Power System," in Transmission and Distribution Conference and Exhibition: Asia and Pacific, 2005 IEEE/PES, Dalian, 2005, pp. 1-7.

[5] I. Dobson, B. A. Carreras, V. E. Lynch, and D. E. Newman, "Complex systems analysis of series of blackouts: Cascading failure, critical points, and self-organization " Chaos: An Interdisciplinary Journal of Nonlinear Science, vol. 17, p. 026103, 2007.

[6] J. Ash and D. Newth, "Optimizing complex networks for resilience against cascading failure," Physica A: Statistical Mechanics and its Applications, vol. 380, pp. 673-683, 2007.

[7] H. Donnell. (2012). Investigation after Auckland rail failure. Available: http://www.nzherald.co.nz/nz/news/article.cfm?c_id=1\&objectid=10801

[8] S. V. Buldyrev, R. Parshani, G. Paul, H. E. Stanley, and S. Havlin, "Catastrophic cascade of failures in interdependent networks," Nature, vol. 464, pp. 1025-1028, 2010.

[9] S. V. Buldyrev, N. W. Shere, and G. A. Cwilich, "Interdependent networks with identical degrees of mutually dependent nodes," Physical Review E, vol. 83, p. 016112, 2011.

[10] R. Parshani, S. V. Buldyrev, and S. Havlin, "Interdependent Networks: Reducing the Coupling Strength Leads to a Change from a First to Second Order Percolation Transition," Physical Review Letters, vol. 105.

[11] J. Shao, S. V. Buldyrev, S. Havlin, and H. E. Stanley, "Cascade of failures in coupled network systems with multiple support-dependence relations," Physical Review E, vol. 83, p. 036116, 2011.

[12] J. Gao, S. V. Buldyrev, S. Havlin, and H. E. Stanley, "Robustness of a Network of Networks," Physical Review Letters, p. 195701, 2011.

[13] P. Bak, C. Tang, and K. Wiesenfeld, "Self-organized criticality: An explanation of the 1/f noise," Physical Review Letters, vol. 59, pp. 381384, 1987.

[14] P. Bak, C. Tang, and K. Wiesenfeld, "Self-organized criticality," Physical Review A, vol. 38, pp. 364-374, 1988.

[15] C. D. Brummitt, R. M. D’Souza, and E. A. Leicht, "Suppressing cascades of load in interdependent networks," Proceedings of the National Academy of Sciences, vol. 109, pp. E680-E689, 2012.

[16] C. Gros, Complex and adaptive dynamical systems : a primer, 2nd ed. Berlin: Springer, 2008.

[17] S. Janson, T. Łuczak, and A. Rucinski, "Random Regular Graphs," in Random Graphs, ed: John Wiley \& Sons, Inc., 2000, pp. 233-270.

[18] M. E. J. Newman, Random graphs. New York: Oxford Inc., 2010

[19] GitHub. (2013). NetwrokX. Available: http://networkx.github.io. 